\title{
Analysis of the added mass term in soil bearing capacity problems
}

\author{
K. A. KWA*, G. D. WEYMOUTH*, D. J. WHITE* and C. M. MARTIN†
}

\begin{abstract}
This technical note presents a simplified method for quantifying the added mass of the soil that is mobilised as part of the failure mechanism around a foundation during rapid loading, and the resulting additional soil resistance. This note focuses on the solutions for an embedded plate anchor, which is a potential foundation system for offshore floating facilities. In current practice, only the shear strength of the soil surrounding the foundation is considered in calculations of the ultimate bearing capacity. However, the solutions presented in this technical note show that the added mass of the soil involved in the failure mechanism around the foundation can result in a significant increase in ultimate bearing capacity during extreme dynamic wave loading events. These lead to snatch loads transmitted to the anchoring and mooring system, which are high but brief. The technical note provides a general approach applicable to all foundation types, and illustrates the effect of the added mass term and the additional capacity for an embedded plate anchor with typical input conditions.
\end{abstract}

KEYWORDS: anchors \& anchorages; bearing capacity; footings/foundations; offshore engineering

Published with permission by the ICE under the CC-BY 4.0 license. (http://creativecommons.org/licenses/by/4.0/)

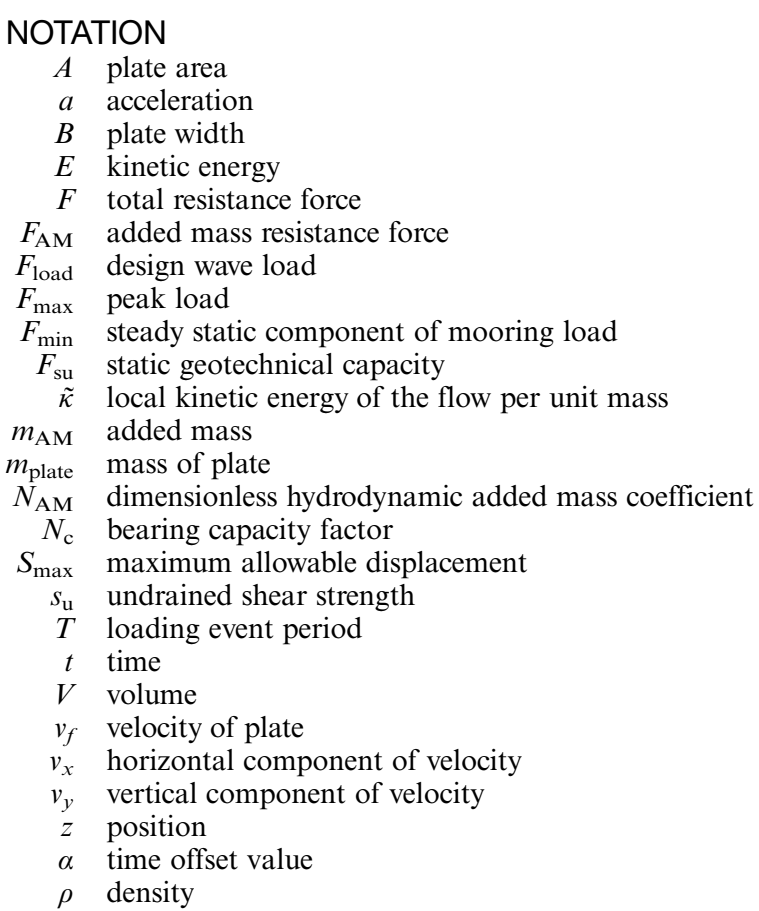

\section{INTRODUCTION}

Offshore renewable energy facilities are extending to floating structures in deeper waters, where stronger wind resources can be harnessed (ORE Catapult, 2018). Meanwhile, floating oil and gas facilities continue to be deployed. Embedded plate anchors are an efficient solution for floating facilities, particularly in soft seabeds. Plate anchors can be installed many ways, including drag-embedment, free-fall,

Manuscript received 17 July 2020; first decision 29 January 2021; accepted 29 January 2021.

Published online at www.geotechniqueletters.com on 9 March 2021.

*University of Southampton, Southampton, UK.

$\dagger$ Department of Engineering Science, University of Oxford, Oxford, UK. screwing or via a suction anchor (Randolph \& Gourvenec, 2017; Aubeny, 2017; O’Loughlin et al., 2017).

The anchor capacity is typically calculated as a multiple of the soil shear strength, with an additional term representing the overburden if the failure mechanism is shallow (Randolph \& Gourvenec 2017; Aubeny, 2017). However, under rapid loading, additional capacity is created from the mass of the soil surrounding the plate. This added mass term is well recognised in fluid mechanics (e.g. Lamb, 1895; Morison et al., 1950; Sarpkaya \& Isaacson, 1981), and is routinely considered in the dynamic motion of floating structures and mooring lines. However, it is ignored in conventional geotechnical capacity analysis.

This technical note documents a method to quantify the added mass of the soil surrounding a plate anchor and the resulting additional resistance. The added mass effect is derived for conventional geotechnical collapse mechanisms in cohesive soils and compared to solutions from classical fluid mechanics. The influence of the contribution from added mass is illustrated for typical input conditions. This study does not consider the additional effects on soil strength $\left(s_{\mathrm{u}}\right)$ relevant to dynamic loading, namely the influence of their rapid and cyclic nature on $s_{\mathrm{u}}$. The cyclic nature may reduce $s_{\mathrm{u}}$, but dynamic loads with higher loading rates may also raise $s_{\mathrm{u}}$ due to the effect of strain rate on strength (Mitchell, 1964, 1993; Koumoto \& Houlsby, 2001; Lunne \& Andersen, 2007; Randolph \& Gourvenec, 2017).

The general approach used in this paper to find the added mass term can also be applied to other materials, where similar effects are evident (e.g. Athani \& Rognon, 2019). It can also be applied to other shapes of foundation - such as piles and caissons - via the same method if the velocity fields mobilised in the soil failure mechanism are identified, for example, experimentally or from a three-dimensional (3D) finite-element analysis.

\section{BACKGROUND}

Geotechnical bearing capacity theory

In undrained soils, the upper-bound theorem is used to define a kinematically admissible collapse mechanism for an embedded plate. Soil flows around a plate moving at a 
velocity $v_{f}$ as shown in Figs 1(a) and 1(b). Hodographs showing the relative velocities throughout the mechanism are shown in Figs 1(c) and 1(d). The internal work dissipation rate on each sliding plane, including the triangular blocks within the shear fan, is the product of the shear forces and relative velocities on the plane. Equating the work input to the dissipation gives the capacity due to the soil strength:

$$
F_{\mathrm{su}}=N_{\mathrm{c}} s_{\mathrm{u}} B
$$

where $N_{\mathrm{c}}$ is the bearing capacity factor, $s_{\mathrm{u}}$ is the soil shear strength and $B$ is the plate width. Solving the work equation yields the same bearing capacity factor for both mechanisms, $N_{\mathrm{c}}=3 \pi+2$ (Meyerhof, 1951; Rowe \& Davis, 1982; Martin \& Randolph, 2001).

Under rapid loading, at soil failure, additional work is required to accelerate the soil mass within the mechanism according to Newton's Second Law. The acceleration varies throughout the mechanism and the net effect is an additional component of resistance $F_{\mathrm{AM}}=m_{\mathrm{AM}} a$ where $a$ is the acceleration of the plate and $m_{\mathrm{AM}}$ is a representative 'added mass'. As described later, this term can also be derived from the collapse mechanism. The total resistance force $(F)$ on the plate after $F_{\text {su }}$ is exceeded is

$$
F=F_{\text {su }}+F_{\mathrm{AM}}
$$

\section{Hydrodynamic added mass theory}

In hydrodynamics, fluid flow around an accelerating plate is a classical problem. The force $\left(F_{\mathrm{AM}}\right)$ due to acceleration of inviscid fluid surrounding an object is analysed by way of the work done by the unsteady reaction forces on a body (Lamb, 1895). For a rigid body moving with speed $v_{f}$, the fluid kinetic energy is defined as

$$
E=\rho v_{f}^{2} \int_{V} \tilde{\kappa} \mathrm{d} V=\frac{1}{2} m_{\mathrm{AM}} v_{f}^{2}
$$

where $\rho$ is the density of the fluid, $V$ is the volume of the fluid influenced by the flow field, $m_{\mathrm{AM}}$ is the lumped added mass term and $\tilde{\kappa}$ is the local kinetic energy of the flow defined per unit mass, normalised by the square of the body velocity. For

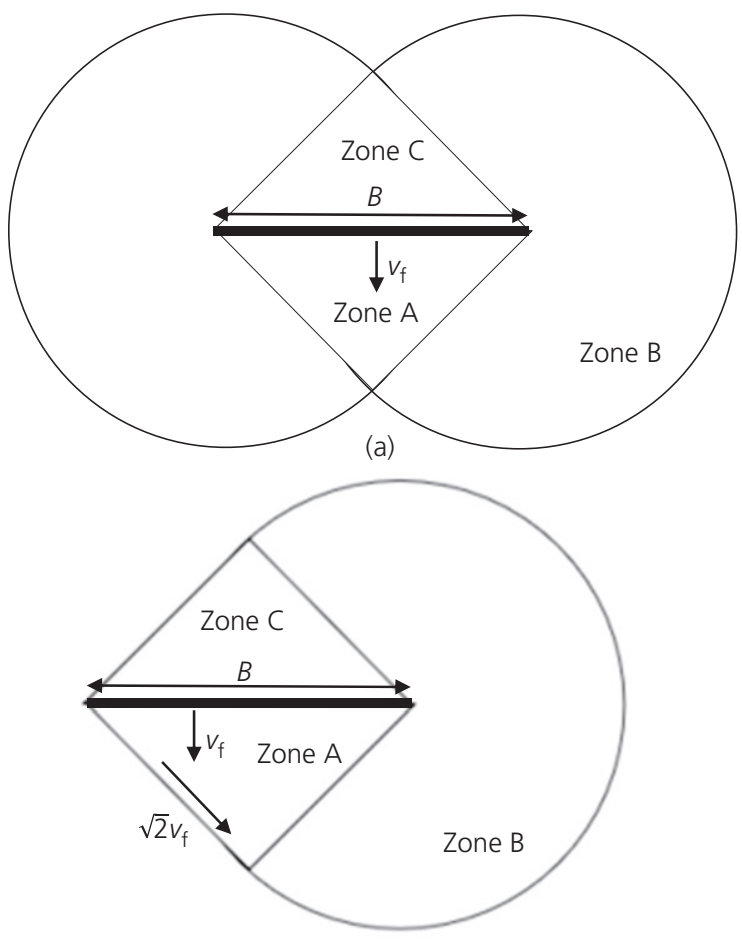

(b)

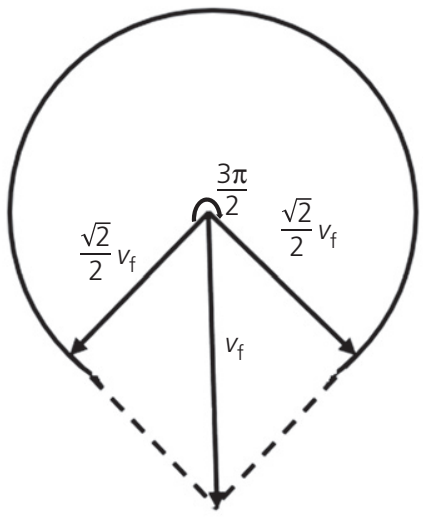

(c)

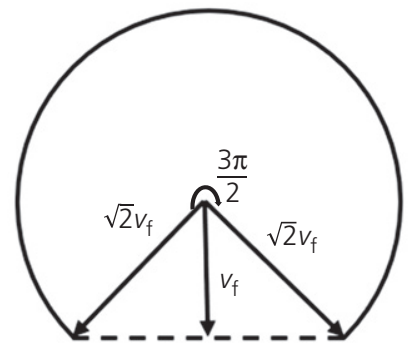

(d)

Fig. 1. Failure mechanism from upper-bound theorem for (a) rough and (b) smooth plates and their accompanying hodographs (c) and (d) 
planar flow, this is

$$
\tilde{\kappa}=\frac{1}{2}\left(\frac{v_{x}^{2}+v_{y}^{2}}{v_{f}^{2}}\right)
$$

The $v_{x}$ and $v_{y}$ terms are the horizontal and vertical component velocities throughout the flow field when the object moves at $v_{f}$. The added mass term can be isolated using equations (3) and (4) as

$$
m_{\mathrm{AM}}=\rho \int_{V} \frac{v_{x}^{2}+v_{y}^{2}}{v_{f}^{2}} \mathrm{~d} V
$$

The added mass term is found by integrating the velocities over the flow field caused the accelerating body and solutions exist for various rigid bodies (Newman, 2018). Using the potential flow solutions for the velocity field, the forces to accelerate a plane strain thin plate, or strip, of width $B$ or a thin circular plate of diameter $B$ in the axisymmetric case, are, respectively:

$$
\begin{aligned}
& F_{\mathrm{AM}}=m_{\mathrm{AM}} a=\frac{\pi}{4} \rho B^{2} a \\
& F_{\mathrm{AM}}=m_{\mathrm{AM}} a=\frac{1}{3} \rho B^{3} a
\end{aligned}
$$

It is useful to define the dimensionless hydrodynamic added mass coefficient as $N_{\mathrm{AM}, 2 \mathrm{D}}=m_{\mathrm{AM}} / \rho B^{2}$ or $N_{\mathrm{AM}, 3 \mathrm{D}}=$ $m_{\mathrm{AM}} / \rho B^{3}$ for the two-dimensional (2D) plane strain and 3D axisymmetric cases - that is, $N_{\mathrm{AM}, 2 \mathrm{D}}=\pi / 4$ and $N_{\mathrm{AM}, 3 \mathrm{D}}=1 / 3$, respectively.

\section{ANALYTICAL SOLUTION FOR $F_{\text {AM }}$}

The same approach as used for fluid flow can be applied to the deformation field defined by the geotechnical upperbound collapse mechanism for the strip plate, so the total force $(F)$ can be written as

$$
F=N_{\mathrm{c}} s_{\mathrm{u}} B+N_{\mathrm{AM}} \rho B^{2} a
$$

The velocities and volume of the sliding blocks within the plane strain collapse mechanism are integrated according to equation (5), for both symmetrical and one-sided Prandtl failure mechanisms (Figures 1(a) and 1(b))

For the symmetrical mechanism, zones $\mathrm{A}$ and $\mathrm{C}$ give equal added mass contributions. The area $(A)$ of the triangles, their velocity terms and resulting added mass contributions, $m_{\mathrm{AM}}$ are

$$
A_{\mathrm{A}, \mathrm{C}}=\frac{B^{2}}{4} ;\left(\frac{v_{x}^{2}+v_{y}^{2}}{v_{f}^{2}}\right)_{\mathrm{A}, \mathrm{C}}=1 ; m_{\mathrm{AM}}^{\mathrm{A}, \mathrm{C}}=\frac{1}{4} \rho B^{2}
$$

In the fan shear zone B, the added mass term from integrating the velocities and areas is

$$
\begin{aligned}
m_{\mathrm{AM}}^{\mathrm{B}}= & \rho \int_{0}^{3 \pi / 2} \frac{1}{v_{f}^{2}}\left(\frac{\sqrt{2}}{2} v_{f}\right)^{2}\left(\sin ^{2}\left(\frac{\pi}{4}+\theta\right)+\cos ^{2}\left(\frac{\pi}{4}+\theta\right)\right) \\
& \times \frac{1}{2}\left(\frac{\sqrt{2}}{2} B\right)^{2} \mathrm{~d} \theta=\frac{3 \pi}{16} \rho B^{2}
\end{aligned}
$$

Therefore, the total added mass term from applying equation (5) over the whole failure mechanism is

$$
m_{\mathrm{AM}}=\left(\frac{1}{2}+\frac{3 \pi}{8}\right) \rho B^{2}=1 \cdot 678 \rho B^{2}
$$

This result is doubled for the one-sided Prandtl mechanism as half of the soil mass is moving at double the speed. The area $(A)$ of the triangles, their velocity terms and resulting added masses in zones $\mathrm{A}$ and $\mathrm{C}$ are

$$
A_{\mathrm{A}, \mathrm{C}}=\frac{B^{2}}{4} ;\left(\frac{v_{x}^{2}+v_{y}^{2}}{v_{f}^{2}}\right)_{\mathrm{A}, \mathrm{C}}=\frac{\left(\sqrt{2} v_{f}\right)^{2}}{v_{f}^{2}}=2 ; m_{\mathrm{AM}}^{\mathrm{A}, \mathrm{C}}=\frac{1}{2} \rho B^{2}
$$

The added mass term in the fan shear zone B is

$$
\begin{aligned}
m_{\mathrm{AM}}^{\mathrm{B}}= & \rho \int_{0}^{3 \pi / 2} \frac{1}{v_{f}^{2}}\left(\sqrt{2} v_{f}\right)^{2}\left(\sin ^{2}\left(\frac{\pi}{4}+\theta\right)+\cos ^{2}\left(\frac{\pi}{4}+\theta\right)\right) \\
& \times \frac{1}{2}\left(\frac{\sqrt{2}}{2} B\right)^{2} \mathrm{~d} \theta=\frac{3 \pi}{4} \rho B^{2}
\end{aligned}
$$

Therefore, the total added mass term is

$$
m_{\mathrm{AM}}=\left(1+\frac{3 \pi}{4}\right) \rho B^{2}=3 \cdot 356 \rho B^{2}
$$

\section{NUMERICAL VERIFICATION OF $F_{\text {AM }}$}

To check equations (10) and (13), and to explore $m_{\mathrm{AM}}$ for a circular plate, numerical analyses of the collapse mechanism were used to create deformation fields to independently determine the added mass term according to equation (5). The numerical analysis utilised OxLim, a developing research software that uses finite-element limit analysis (FELA) to solve geotechnical lower- and upper-bound solutions (Makrodimopoulos \& Martin, 2006, 2007, 2008). Four cases were considered; rough and smooth plates, with plane strain (strip) and circular (disc) shapes. The soil had homogenous undrained shear strength and unit weight.

The resulting bearing capacity factors obtained with the Tresca failure criterion matched to within $0 \cdot 1 \%$ the exact plasticity solutions (Martin \& Randolph 2001). The added mass coefficients were found from equation (5), by integrating the velocities and volume of each element over the flow field within the full soil domain. For the plane strain case $m_{\mathrm{AM}}$ was within $2 \%$ of the analytical solution for the symmetrical mechanism (equations (8)-(10)) (Table 1). The added mass coefficient for the circular plate was lower than the plane strain case by a factor of $\sim 2$. This reflects the more localised failure mechanism, so less mass is accelerated (Table 1).

\section{SUMMARY AND APPLICATIONS \\ Summary of bearing and added mass coefficients}

Tables 1 and 2 summarise the bearing capacity factors $\left(N_{\mathrm{c}}\right)$ and added mass coefficients $\left(N_{\mathrm{AM}}\right)$ determined using the fluid mechanics and geotechnical approaches. For the inviscid flow solution, an equivalent bearing capacity factor was determined by developing the work equation analytically (see Appendix).

$N_{\text {AM }}$ from the geotechnical collapse mechanism is significantly larger than from the inviscid flow field because of the different flow mechanisms. In inviscid fluids, there is no shear resistance within the material and the flow field develops such that the added mass term is minimised. However, in soil, with shear resistance, the optimal flow field minimises the dissipation. The resulting mechanism is larger, resulting in a higher added mass. 
Table 1. Summary of bearing capacity factors $\left(N_{C}\right)$ and added mass coefficients $\left(N_{\mathrm{AM}, 2 \mathrm{D}}\right)$ for a plane strain plate

\begin{tabular}{l|c|c}
\hline Method & $N_{\mathrm{c}}$ & $N_{\mathrm{AM}, 2 \mathrm{D}}$ \\
\hline Fluid inviscid flow field & $13 \cdot 750$ & $0 \cdot 785$ \\
Geotechnical analytical UB* & $11 \cdot 425$ & $1 \cdot 678 \dagger, 3 \cdot 356 \dagger$ \\
Geotechnical numerical UB (FELA) & $11 \cdot 439+, 11 \cdot 431 \dagger$ & $1 \cdot 648$ \\
\hline
\end{tabular}

*Meyerhof (1951).

$\$$ Rough and smooth analyses.

$†$ Prandtl symmetrical and one-sided mechanisms.

Table 2. Summary of bearing capacity factors $\left(N_{C}\right)$ and added mass coefficients $\left(N_{\mathrm{AM}, 3 \mathrm{D}}\right)$ for an axisymmetric disc

\begin{tabular}{|c|c|c|}
\hline Method & $N_{\mathrm{c}}$ & $N_{\mathrm{AM}, 3 \mathrm{D}}$ \\
\hline $\begin{array}{l}\text { Fluid inviscid flow field } \\
\text { Geotechnical analytical UB } \dagger \\
\text { Geotechnical numerical UB (FELA) }\end{array}$ & $\begin{array}{l}16 \cdot 198^{*}, 14 \cdot 842^{*} \\
13 \cdot 11 \ddagger, 12 \cdot 42 \$ \\
13 \cdot 13 \ddagger, 12 \cdot 435 \$\end{array}$ & $0.333-$ \\
\hline
\end{tabular}

*Tresca, Von Mises.

$\dagger$ Martin \& Randolph (2001).

$\$$ Rough and smooth analyses.

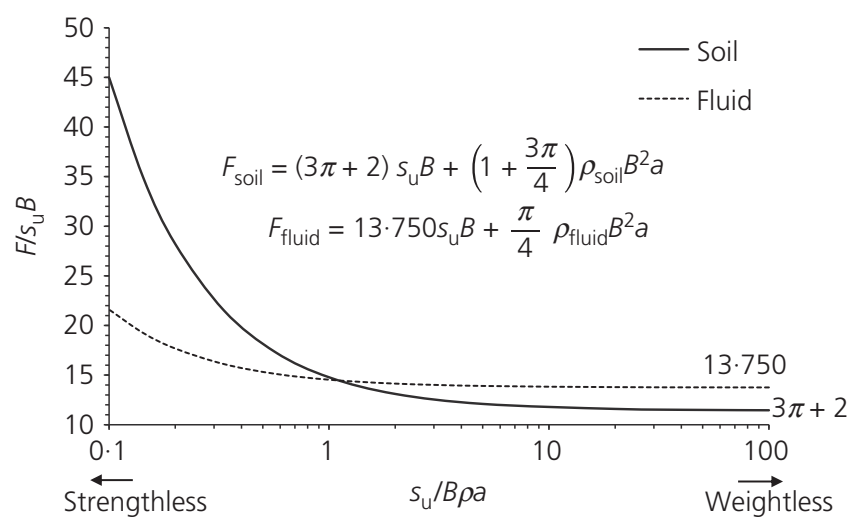

(a)

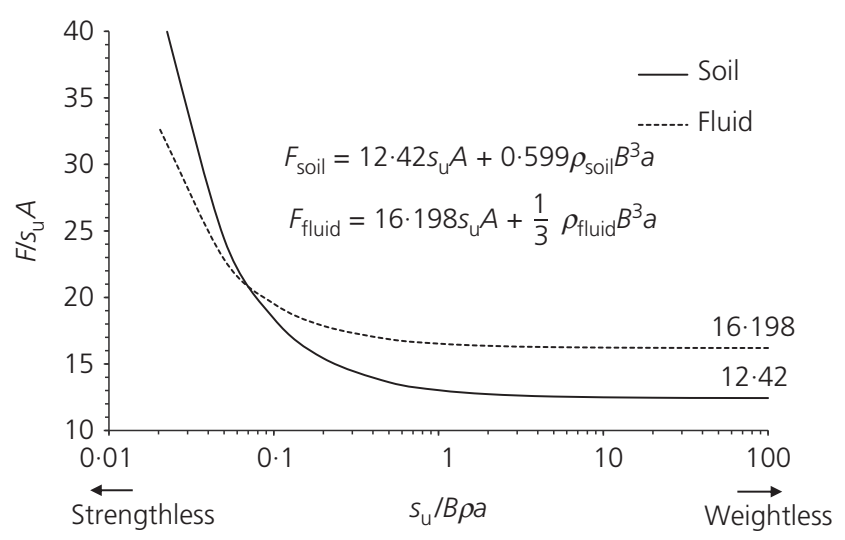

(b)

Fig. 2. Variations in the contributions of shear strength and the added mass of the material, with respect to the dimensionless group $s_{\mathrm{u}} / B \rho a$ for (a) smooth plane strain plate and (b) smooth circular disc

These failure mechanisms that are exact for inviscid flow and rigid-plastic flow represent extremes of the soil response across the potential range of loading rates. The different contributions of the shear strength and added mass to the total resistance are shown in Fig. 2 across the range of dimensionless group $s_{\mathrm{u}} / B \rho a$, which represents the balance between strength and inertia forces.

When $s_{\mathrm{u}} / B \rho a$ is high, the shear strength term dominates, with the soil weightless in the limit $s_{\mathrm{u}} / B \rho a \rightarrow \infty$ so the uplift capacity approaches the rigid-plastic solution. Conversely, at low $s_{\mathrm{u}} / B \rho a$, the added mass term dominates and in the limit the soil is strengthless.

The combined resistance from the bearing capacity and added mass coefficients of the inviscid and rigid-plastic solutions are consistent with this transition. At high $s_{\mathrm{u}} / B \rho a$ the rigid-plastic mechanism offers lower total resistance and therefore the optimal failure mechanism, whereas at low $s_{\mathrm{u}} / B \rho a$ the inviscid flow solution is lower. The transition is at $s_{\mathrm{u}} / B \rho a \sim 1$ for plane strain, and $\sim 0.08$ for axisymmetry. In practice, the optimal mechanism that is mobilised could involve a mixture of the rigid-plastic and inviscid flow fields, which are superposable. The transition between rigid - plastic, inviscid flow, or a mixture, is likely to be smooth, similar to experimental observations for steady flow around a cylinder in soft soil (Sahdi et al., 2014).

\section{Illustrative example}

This example shows the influence of added mass for a typical design event of a force impulse $\left(F_{\text {load }}\right)$ representing a snatch loading from a wave. Snatch loads are caused by dynamic mooring effects and can have a period $\sim 10$ times shorter than the wave itself (e.g. Hann et al., 2015; Lind et al., 2016). In geotechnical design, failure of a plate anchor can be defined at a displacement $\left(S_{\max }\right)$ of $10 \%$ of the plate diameter.

The impulse, $F_{\text {load }}$, is represented by (Fig. 3)

$$
F_{\text {load }}=F_{\text {min }}+\left(F_{\max }-F_{\min }\right)\left(\frac{\sin ((t-\alpha) / T)}{(t-\alpha) / T}\right)
$$

where $F_{\text {min }}$ represents a steady static component of the mooring load and $F_{\max }$ is the peak load, varying with time, $t$, offset by $\alpha$, with $T$ being the event period. When $F_{\text {load }}$ exceeds $F_{\text {su }}$ the plate moves and Newmark's $\beta$ method is used to calculate the resulting response (Fig. 4). Therefore, the response of the plate, when $F_{\text {load }}>F_{\text {su }}$, is defined by the following equation of motion:

$$
\sum F=F_{\text {load }}-F_{\text {su }}-F_{\text {AM }}=m_{\text {plate }} a
$$




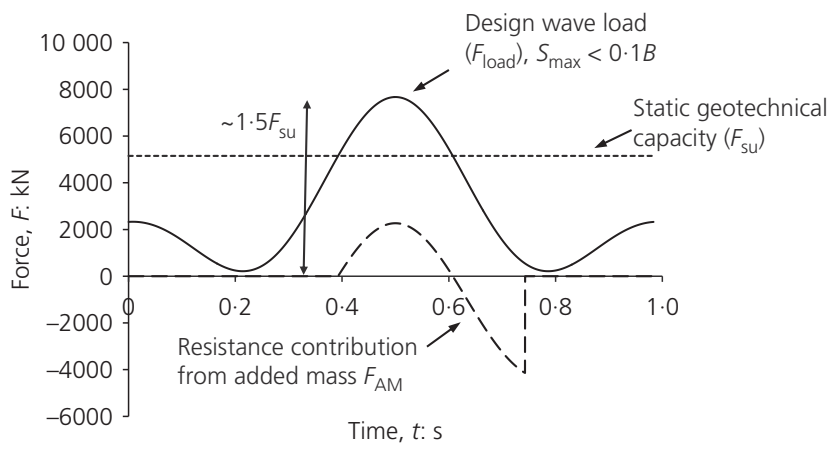

Fig. 3. Forces acting on embedded plate anchor during impulse snatch load

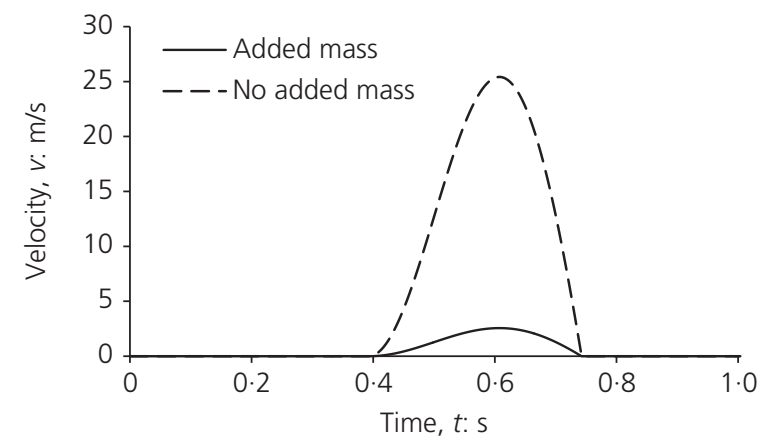

(a)

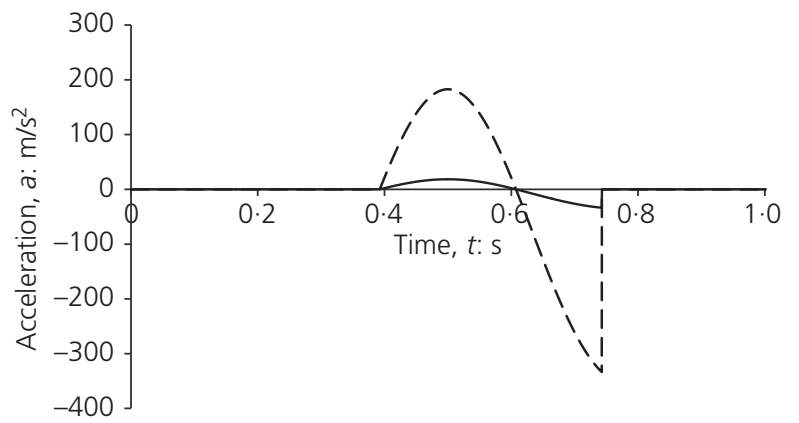

(b)

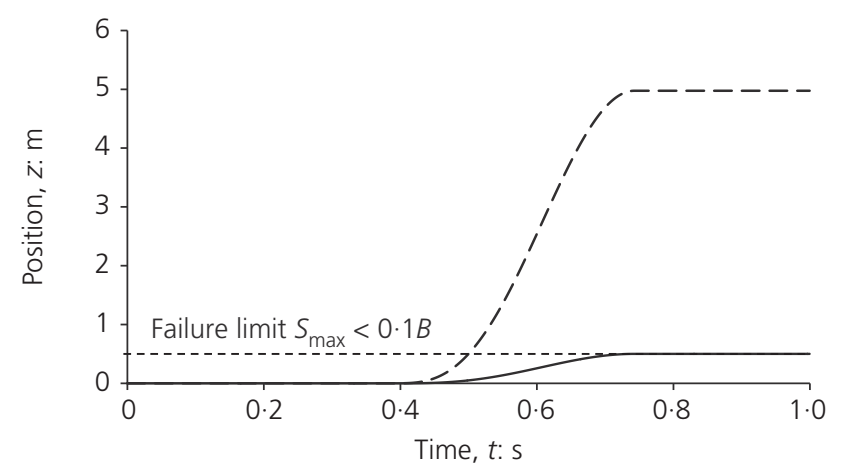

(c)

Fig. 4. Anchor movements during impulse snatch load with and without added mass effects

where $m_{\text {plate }}$ is the mass of the plate. In Fig. $3, F_{\mathrm{AM}}$ is the extra resistance force from the acceleration of the soil mass involved in the failure mechanism during the impulse load. The anchoring system withstands a peak load that is $\sim 1.5$ times greater than the geotechnical static capacity, due to the added mass contribution, without exceeding the
Table 3. Summary of parameters used in the illustrative example

\begin{tabular}{l|l|l}
\hline Input parameters & Value & Units \\
\hline $\begin{array}{l}\text { Plate properties } \\
\text { Plate diameter, } B\end{array}$ & 5 & $\mathrm{~m}$ \\
$\begin{array}{l}\text { Soil properties } \\
\text { Soil density, } \rho\end{array}$ & 1800 & $\mathrm{~kg} / \mathrm{m}^{3}$ \\
Undrained shear strength $s_{\mathrm{u}}$ & 20 & $\mathrm{kPa}$ \\
$\begin{array}{l}\text { Geotechnical capacity } \\
\text { Bearing factor, } N_{\mathrm{c}}\end{array}$ & $13 \cdot 11$ & - \\
$\begin{array}{l}\text { Geotechnical capacity } F_{\mathrm{su}} \\
\text { Added mass resistance } \\
\text { Added mass coefficient } N_{\mathrm{AM}}\end{array}$ & $5128 \cdot 65$ & $\mathrm{kN}$ \\
Added mass & $0 \cdot 548$ & - \\
\hline
\end{tabular}

allowable displacement of $S_{\max }=0 \cdot 1 B$ (Fig. 4(c)). This example shows that the added mass contribution can significantly enhance the capacity available to resist dynamic loads, beyond the geotechnical capacity typically used in practice. It is possible that the added mass, which is overlooked in design, is partly responsible for mooring systems failures being primarily due to mooring lines breaking, rather than anchors pulling out (Ma et al., 2019).

A second dynamic response for the same impulse load is also shown in Fig. 4, for a case in which the mass of the plate is considered, but not the added soil mass. In this case the plate displacement from the loading event is $>5 \mathrm{~m}$, approximately 10 times more than when the soil added mass is included (Fig. 4(c)). This is consistent with the added mass being 10 times greater than the mass of the plate itself. A buried plate is efficient in this respect compared to other foundation types such as a vertically loaded pile, for which the failure mechanism mobilises much less added mass. A summary of the parameters and values used in this illustrative example is included in Table 3.

\section{CONCLUDING REMARKS}

This technical note has documented a simple method for determining the added mass resistance during dynamic loading events on embedded foundations, utilising conventional geotechnical collapse mechanisms. These solutions are a geotechnical counterpart to the established added mass solutions derived for fluid flow. The solutions provide the additional contribution to the geotechnical resistance from the mass of soil accelerated in the failure mechanism during dynamic loading. An illustrative example shows that this additional resistance can significantly enhance the foundation capacity during dynamic loading events such as snatch loads within a mooring system. This general approach is not limited to embedded plate foundations but applies to other foundations, including shallow foundations or piles that are dynamically loaded. It provides a basis to quantify a potentially large additional component of geotechnical resistance that is overlooked in conventional design.

\section{ACKNOWLEDGEMENTS}

The authors acknowledge support from the EPSRC Offshore Renewable Energy Supergen hub (grant EPSRC $\mathrm{EP} / \mathrm{S} 000747 / 1)$.

\section{APPENDIX}

To find an upper-bound plasticity solution for a failure mechanism or flow field, the internal work rate, $W_{i}^{V}$ as an object moves through a continuum field of volume $V$ with a 
constant undrained strength $s_{\mathrm{u}}$ is

$$
W_{i}^{V}=s_{\mathrm{u}} \int_{V} \dot{\gamma}_{\max }, N_{\mathrm{c}}=\frac{W_{i}^{V}}{A U s_{\mathrm{u}}}
$$

where $N_{\mathrm{c}}$ is the work factor scaled by the anchor frontal area $A$ and normal velocity $U$, and $\dot{\gamma}_{\text {max }}$ is the maximum local strain rate defined in terms of principal components or eigenvalues, $\lambda_{i}$ of the infinitesimal strain rate tensor $D$

$$
D=\frac{1}{2}\left(\nabla u+\nabla u^{\mathrm{T}}\right)
$$

where $u$ is the continuum velocity. As $D$ is real and symmetric, it has real eigenvalues which can be found analytically in terms of the tensor $\operatorname{trace} \operatorname{tr}(D)=\sum \lambda_{i}$ and determinant $\operatorname{det}(D)=\prod \lambda_{i}$. In particular, the maximum strain rate for the Tresca condition is given by $\dot{\gamma}_{\max }=$ $2 \max |\lambda|=2 \max \left(\lambda_{1},-\lambda_{3}\right)$ where $\lambda_{1}>\lambda_{2}>\lambda_{3}$. For the Von Mises condition, $\dot{\gamma}_{\max }=\sqrt{2 \sum \lambda_{i}^{2}}$. Dissipation due to the relative velocity at the object surface is neglected, which corresponds to the smooth case in the geotechnical analysis.

\section{Incompressible irrotational $2 D$ and axisymmetric flow}

If the velocity field is irrotational, it can be defined by a potential function $u=\nabla \phi$ meaning $D=H \phi$, where $H$ is the Hessian matrix. If the flow is also incompressible then $\nabla$. $u=0$ and the potential satisfies the Laplace equation $\nabla^{2} \phi=\operatorname{tr}(D)=0$. Classical solutions for many flow potentials are known, such as from Lamb (1895); however, the Hessian of a potential and its eigenvalues have not been well studied. If a $3 \mathrm{D}$ flow defined in coordinates $q_{1}, q_{2}, q_{3}$ is $2 \mathrm{D}$ or axisymmetric about the coordinate $q_{3}$ then $\partial \phi / \partial q_{3}=0$ and the strain rate matrix takes the general form

$$
D=H(\phi)=\left[\begin{array}{ccc}
D_{11} & D_{12} & 0 \\
D_{12} & D_{22} & 0 \\
0 & 0 & D_{33}
\end{array}\right]
$$

where $D_{33}=-D_{11}-D_{22}$ due to incompressibility. Since the $q_{3}$ rows and columns are linearly independent there is a trivial mean eigenvalue $\lambda_{2}=D_{33}=-D_{11}-D_{22}$. With that eigenvalue known, the characteristic equation is reduced to a quadratic and the other eigenvalues are

$$
\lambda_{1,3}=\frac{1}{2}\left[-\lambda_{2} \pm \sqrt{\lambda_{2}^{2}-4 \operatorname{det}\left(D_{2 d}\right)}\right]
$$

where $\operatorname{det}\left(D_{2 d}\right)=D_{11} D_{22}-D_{12}^{2}$. Substitution gives the axisymmetric maximum strain rate for the two failure criteria as

$$
\dot{\gamma}_{\max }=\left\{\begin{array}{cc}
\left|\lambda_{2}\right|+\sqrt{\lambda_{2}^{2}-4 \operatorname{det}\left(D_{2 d}\right)}, & \text { Tresca } \\
2 \sqrt{\lambda_{2}^{2}-\operatorname{det}\left(D_{2 d}\right)}, & \text { Von Mises }
\end{array}\right.
$$

For fully $2 \mathrm{D}$ flow we must also have $D_{33}=\lambda_{2}=0$ and so $\dot{\gamma}_{\text {max }}=2 \sqrt{-\operatorname{det}\left(D_{2 d}\right)}$ for either failure criterion.

\section{Flows using complex variables}

For 2D potential flow with a holomorphic complex potential $\Phi(\zeta)$, where $\zeta=x+\mathrm{i} y=r \mathrm{e}^{\mathrm{i} \theta}, \mathrm{d} \Phi / \mathrm{d} \zeta=\mathrm{d} \Phi / \mathrm{dx}=$ $\mathrm{d} \Phi / \mathrm{d}(\mathrm{i} y)$. This means the strain rate tensor and maximum strain rate are

$$
D_{x x}=-D_{y y}=\operatorname{Re}\left|\Phi^{\prime \prime}\right|, D_{x y}=-\operatorname{Im}\left|\Phi^{\prime \prime}\right|, \dot{\gamma}_{\max }=2\left|\Phi^{\prime \prime}\right|
$$

Complex variables make it simple to map any potential $\Phi$ to a new coordinate system using a transformation function $\chi=f(\zeta)$. Using the chain rule, the complex velocity is

$$
w=u_{x}-\mathrm{i} u_{y}=\frac{\mathrm{d} \Phi}{\mathrm{d} \chi}=\frac{\Phi^{\prime}}{\chi^{\prime}}
$$

where $\chi^{\prime}=\mathrm{d} \chi / \mathrm{d} \zeta$. Applying the product and chain rule again to give the second derivative and strain rate as

$$
\frac{\mathrm{d}^{2} \Phi}{\mathrm{d} \chi^{2}}=\frac{\mathrm{d}}{\mathrm{d} \chi}\left(\frac{\Phi^{\prime}}{\chi^{\prime}}\right)=\frac{\Phi^{\prime \prime}-\Phi^{\prime} \chi^{\prime \prime} / \chi^{\prime}}{\chi^{\prime 2}} \rightarrow \dot{\gamma}_{\max }=\frac{2}{J}\left|\Phi^{\prime \prime}-\Phi^{\prime} \frac{\chi^{\prime \prime}}{\chi^{\prime}}\right|
$$

where $J=|\mathrm{d} \chi / \mathrm{d} \zeta|^{2}$ is the Jacobian. The volume integral over $V$ will also be adjusted in the transformed space as $\mathrm{d} V=\mathrm{d} \xi \mathrm{d} \varphi=J \mathrm{~d} x \mathrm{~d} y$. Therefore, the Jacobians cancel and the work per unit depth is

$$
W_{i}^{v}=s_{u} \int_{V} 2\left|\Phi^{\prime \prime}-\Phi^{\prime} \frac{\chi^{\prime \prime}}{\chi^{\prime}}\right| \mathrm{d} x \mathrm{~d} y
$$

\section{General elliptical cylinder}

The circle defined by $\zeta=a \mathrm{e}^{\mathrm{i} \theta}$ is mapped to an ellipse using the transformation $\chi=\zeta-\varepsilon a^{2} \zeta^{-1}$. The area (per unit length) of the resulting ellipse is $A=2 a(1+\varepsilon)$ and the aspect ratio is $\mathrm{AR}=(1-\varepsilon) /(1+\varepsilon)$, so setting $\varepsilon=0$ recovers a circle and $\varepsilon=1$ results in a flat plate. Lamb (1895) shows a dipole $\Phi=k \zeta^{-1}$ describes the flow induced by this moving ellipse, where $k$ is the dipole strength. Computing $\Phi^{\prime}=-k \zeta^{-2}$ and $\chi^{\prime}=1+\varepsilon a^{2} \zeta^{-2}$, the complex velocity is

$$
w=-\frac{k \zeta^{-2}}{1+\varepsilon a^{2} \zeta^{-2}}
$$

Therefore, $k=U a^{2}(1+\varepsilon)$ to achieve surface velocity $w=-U$ when $r=a, \theta=0, \pi$. Note the velocity is singular at $\varepsilon=1, r=a, \theta= \pm \pi / 2$, corresponding to the corners of a flat plate. Computing $\Phi^{\prime \prime}=2 k \zeta^{-3}$ and $\chi^{\prime \prime}=-2 \varepsilon a^{2} \zeta^{-3}$ and substituting gives the second derivative as

$$
\frac{\mathrm{d}^{2} \Phi}{\mathrm{d} \chi^{2}}=\frac{2 k \zeta^{-3}}{\left(1+\varepsilon a^{2} \zeta^{-2}\right)^{3}}
$$

From which the components of $D$ and $\dot{\gamma}_{\max }$ are determined. Finally, the work rate for a general elliptical cylinder is then

$$
\begin{aligned}
W_{i}^{v} & =2 s_{\mathrm{u}} \int_{0}^{2 \pi} \int_{a}^{\infty}\left|\frac{2 k \zeta^{-3}}{\left(1+\varepsilon a^{2} \zeta^{-2}\right)^{3}}\right| r \mathrm{~d} r \mathrm{~d} \theta \\
& =16 k s_{\mathrm{u}} \int_{0}^{\pi / 2} \int_{a}^{\infty} \frac{\mathrm{d} r \mathrm{~d} \theta}{\mid r^{2}+\varepsilon a^{2} \mathrm{e}^{-2 i \theta \mid}}
\end{aligned}
$$

which gives $N_{\mathrm{c}}=4 \pi$ for a circle $(\varepsilon=0)$, as in Einav and Randolph (2005) and $N_{\mathrm{c}} \approx 13.750$ for the plate. The full range of integration can be determined numerically and the result is summarised in Fig. 5.

\section{Sphere}

In axisymmetric flow, we cannot use the complex variable coordinate system, but choosing coordinates aligned with the body geometry ensures $\phi=Q_{1}\left(q_{1}\right) Q_{2}\left(q_{2}\right)$. For example, the flow induced by a sphere is described by the potential $\phi=1 / 2 U a^{3} r^{-2} \cos \theta=R(\mathrm{r}) \Theta(\theta)$. Note the axisymmetric variable $q_{3}=\varphi$ does not appear. The strain tensor components are more complex due to the metrics not 


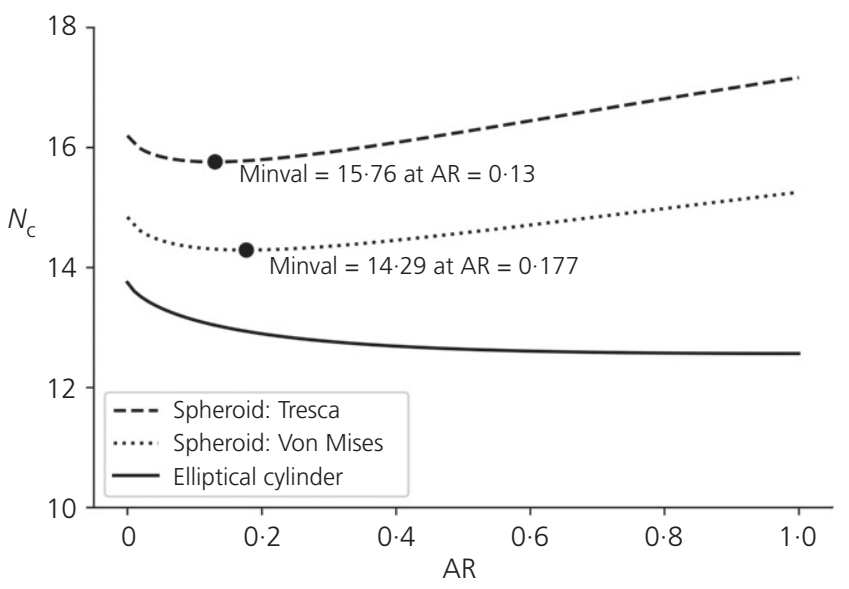

Fig. 5. Variation of the cylinder and spheroid work rate integral with changing aspect ratio

being constant, and are given by

$$
\begin{aligned}
& D_{r r}=R^{\prime \prime} \Theta=2 \beta \cos \theta \\
& D_{\theta \theta}=\frac{R^{\prime}}{r} \Theta+\frac{R}{r^{2}} \Theta^{\prime \prime}=-\beta \cos \theta \\
& D_{r \theta}=\left[\frac{R^{\prime}}{r}-\frac{R}{r^{2}}\right] \Theta=\beta \sin \theta
\end{aligned}
$$

where $\beta=3 U a^{3} / 2 r^{4}$. As such, we have $\lambda_{2}=-\beta \cos \theta$ and $\operatorname{det}\left(D_{2 D}\right)=-\beta^{2}\left(2 \cos ^{2} \theta+\sin ^{2} \theta\right)$ and so

$$
\frac{\dot{\gamma}_{\max }}{\beta}=\left\{\begin{array}{cc}
|\cos \theta|+\sqrt{5 \cos ^{2} \theta+4}, & \text { Tresca } \\
2 \sqrt{2 \cos ^{2} \theta+1}, & \text { Von Mises }
\end{array}\right.
$$

Integration gives the work integral as

$$
\begin{array}{cl}
W_{i}^{V}=2 \pi \int_{0}^{\pi} \int_{0}^{\infty} \dot{\gamma}_{\max } r^{2} \sin \theta \mathrm{d} r \mathrm{~d} \theta \rightarrow & \\
\frac{W_{i}^{V}}{A U s_{\mathrm{u}}}=3 \int_{0}^{\pi} \frac{\dot{\gamma}_{\max }}{\beta} \sin \theta \mathrm{d} \theta \approx \begin{cases}17 \cdot 165, & \text { Tresca } \\
15 \cdot 255, & \text { Von Mises }\end{cases}
\end{array}
$$

where $A=\pi a^{2}$ is the frontal area.

\section{Oblate spheroid coordinates}

Obtaining solutions for more general sphere-like shapes requires a more cumbersome coordinate system such as oblate spheriodal coordinates $\zeta, \mu, \varphi$ defined as

$$
x=a \mu \zeta, y=\rho \cos \varphi, z=\rho \sin \varphi, \rho^{2}=a^{2}\left(1-\mu^{2}\right)\left(1+\zeta^{2}\right)
$$

where $0 \leq \zeta<\infty,-1 \leq \mu \leq 1$ and $a$ is the focal radius.

The scale factors and Jacobian are

$$
\begin{aligned}
h_{\zeta} & =a \sqrt{\frac{\zeta^{2}+\mu^{2}}{1+\zeta^{2}}}, h_{\mu}=a \sqrt{\frac{\zeta^{2}+\mu^{2}}{1-\mu^{2}}}, h_{\varphi}=\rho, \\
J & =a^{3}\left(\zeta^{2}+\mu^{2}\right)
\end{aligned}
$$

Finally, the independent components of the strain matrix applied to an axisymmetric field $\phi=Z(\zeta) M(\mu)$ are

$$
D_{\zeta \zeta}=\frac{M}{h_{\zeta}} \frac{\partial}{\partial \zeta} \frac{Z^{\prime}}{h_{\zeta}}+\frac{Z M^{\prime}}{h_{\zeta} h_{\mu}^{2}} \frac{\partial h_{\zeta}}{\partial \mu}=\frac{Z^{\prime \prime} M}{h_{\zeta}^{2}}+\frac{\mu Z M^{\prime}-\zeta Z^{\prime} M}{h_{\mu}^{2}\left(\zeta^{2}+\mu^{2}\right)}
$$

$$
\begin{aligned}
D_{\mu \mu} & =\frac{Z}{h_{\mu}} \frac{\partial}{\partial \mu} \frac{M^{\prime}}{h_{\mu}}+\frac{Z^{\prime} M}{h_{\mu} h_{\zeta}^{2}} \frac{\partial h_{\mu}}{\partial \zeta}=\frac{Z M^{\prime \prime}}{h_{\mu}^{2}}+\frac{\zeta Z^{\prime} M-\mu Z M^{\prime}}{h_{\zeta}^{2}\left(\zeta^{2}+\mu^{2}\right)} \\
D_{\zeta \mu} & =\frac{h_{\mu} M^{\prime}}{2 h_{\zeta}} \frac{\partial}{\partial \zeta} \frac{Z}{h_{\mu}^{2}}+\frac{h_{\zeta} Z^{\prime}}{2 h_{\mu}} \frac{\partial}{\partial \mu} \frac{M}{h_{\zeta}^{2}} \\
& =\frac{Z^{\prime} M^{\prime}}{h_{\zeta} h_{\mu}}-\frac{\zeta Z M^{\prime}+\mu Z^{\prime} M}{h_{\zeta} h_{\mu}\left(\zeta^{2}+\mu^{2}\right)}
\end{aligned}
$$

Disc

A disc of radius $a$ is obtained on $\zeta=0$ and the flow potential induced by perpendicular motion of this disc is given by $=(2 U a / \pi) \mu\left(1-\zeta \cot ^{-1} \zeta\right)(\mathrm{Lamb}, 1895)$. Therefore,

$$
Z=1-\zeta \cot ^{1} \zeta, Z^{\prime}=\frac{\zeta}{1+\zeta^{2}}-\cot ^{1} \zeta, Z^{\prime \prime}=\frac{2}{\left(1+\zeta^{2}\right)^{2}}
$$

$$
M=\frac{2 U a}{\pi} \mu, M^{\prime}=\frac{2 U a}{\pi}, M^{\prime \prime}=0
$$

Substituting these into the oblate strain matrix formula and integrating over the oblate coordinates gives

$$
\frac{W_{i}^{V}}{\pi a^{2} U s_{u}} \approx \begin{cases}16 \cdot 198, & \text { Tresca } \\ 14 \cdot 842, & \text { Von Mises }\end{cases}
$$

Which are quite similar to the sphere results. Figure 5 shows the value of scaled work as a function of the oblate spheroid AR (adjusted by using different $\zeta$ surfaces). The work is fairly constant, but is at a minimum for spheroids around $\mathrm{AR} \sim 0 \cdot 15$.

\section{REFERENCES}

Athani, S. \& Rognon, P. (2019). Inertial drag in granular media. Phys. Rev, Fluids 4, No. 12, 124302.

Aubeny, C. (2017). Geomechanics of marine anchors. Boca Raton, FL, USA: CRC Press.

Einav, I. \& Randolph, M. F. (2005). Combining upper bound and strain path methods for evaluating penetration resistance. Int. J. Numer. Methods Engng 63, No. 14, 1991-2016.

Hann, M., Greaves, D. \& Raby, A. (2015). Snatch loading of a single taut moored floating wave energy converter due to focussed wave groups. Ocean Engng 96, 258-271.

Koumoto, T. \& Houlsby, G. T. (2001). Theory and practice of the fall cone test. Géotechnique 51, No. 8, 701-712, https://doi.org/ 10.1680/geot.2001.51.8.701.

Lamb, H. (1895). Hydrodynamics. London, UK: Cambridge University Press.

Lind, S. J., Stansby, P. K. \& Rogers, B. D. (2016). Fixed and moored bodies in steep and breaking waves using SPH with the FroudeKrylov approximation. J. Ocean Engng Marine Energy 2, No. 3, 331-354.

Lunne, T. \& Andersen, K. H. (2007). Soft clay shear strength parameters for deepwater geotechnical design. In Offshore site investigation and geotechnics: confronting new challenges and sharing knowledge. London, UK: Society of Underwater Technology.

Ma, K. T., Luo, Y., Kwan, T. \& Wu, Y. (2019). Mooring system reliability. Chapter 13 in mooring system engineering for offshore structures, pp. 255-279. Cambridge, MA, USA and Oxford, UK: Gulf Professional Publishing.

Makrodimopoulos, A. \& Martin, C. M. (2006). Lower bound limit analysis of cohesive-frictional materials using second-order cone programming. Int. J. Numer. Methods Engng 66, No. 4, $604-634$.

Makrodimopoulos, A. \& Martin, C. M. (2007). Upper bound limit analysis using simplex strain elements and second-order cone 
programming. Int. J. Numer. Anal. Methods Geomech. 31, No. 6, 835-865.

Makrodimopoulos, A. \& Martin, C. M. (2008). Upper bound limit analysis using discontinuous quadratic displacement fields. Commun. Numer. Methods Engng 24, No. 11, 911-927.

Martin, C. M. \& Randolph, M. F. (2001). Applications of the lower and upper bound theorems of plasticity to collapse of circular foundations. In Proc. 10th Int. Conf. on Computer Methods and Advances in Geomechanics, vol. 2, pp. 1417-1428. Abingdon, UK: Taylor \& Francis.

Meyerhof, G. G. (1951). The ultimate bearing capacity of foundations. Géotechnique 2, No. 4, 301-332, https://doi.org/ 10.1680/geot.1951.2.4.301.

Mitchell, J. K. (1964). Shearing resistance of soils as a rate process. J. Soil Mech. Found. Div. 90, No. SM 1, 29-61.

Mitchell, J. K. (1993). Fundamentals of soil behaviour, 2nd edn. New York, NY, USA: John Wiley and Sons.

Morison, J. R., O’Brien, M. P., Johnson, J. W. \& Schaaf, S. A. (1950). The force exerted by surface waves on piles. Petrol. Trans. 2, No. 5, 149-154, https://doi.org/10.2118/950149-G

Newman, J. N. (2018). Marine hydrodynamics. Cambridge, MA, USA and London, UK: MIT Press.
O'Loughlin, C. D., White, D. J. \& Stanier, S. A. (2017). Plate anchors for mooring floating facilities - a view towards unlocking cost and risk benefits. In Offshore site investigation geotechnics Proceedings of the 8th international conference proceeding, vol. 978, No. 986, pp. 978-986. Perth, Australia: Society for Underwater Technology.

ORE Catapult (2018). Report: macroeconomic benefits of floating offshore wind in the UK. Report for the Crown Estate Scotland. Glasgow, UK: ORE Catapult.

Randolph, M. \& Gourvenec, S. (2017). Offshore geotechnical engineering. Oxford, UK and New York, NY, USA: Spon Press, Taylor \& Francis.

Rowe, R. K. \& Davis, E. H. (1982). The behaviour of anchor plates in clay. Géotechnique 32, No. 1, 9-23, https://doi.org/10.1680/ geot.1982.32.1.9.

Sahdi, F., Gaudin, C., White, D. J., Boylan, N. P. \& Randolph, M. F. (2014). Centrifuge modelling of active slide-pipeline loading in soft clay. Géotechnique 64, No. 1, 16-27, https://doi.org/10.1680/ geot.12.P.191.

Sarpkaya, T. \& Isaacson, M. (1981). Mechanics of wave forces on offshore structures. New York, NY, USA: Van Nostrand Reinhold, ISBN: 0-442-25402-4. 